\title{
Influência da luminosidade no crescimento e floração de Dendrobium nobile Lindl. ${ }^{(1)}$
}

\author{
YARA BRITO CHAIM JARDIM ROSA(2), FERNANDA ZANDONADI RAMOS(3), ROSANGELA GUARISSO DE \\ SOUZA $^{(4)}$, JACKELINE SCHULTZ SOARES(5), EDGARD JARDIM ROSA JUNIOR(2), NILDA TIYOKO KOBAYASHI \\ HOFFMANN(5), DEREK BRITO CHAIM JARDIM ROSA(6) e JOSÉ CARLOS SORGATO(6)
}

\begin{abstract}
RESUMO
Este estudo, conduzido na área de Jardinocultura da Faculdade de Ciências Agrárias da UFGD de setembro de 2010 a agosto de 2011, teve por objetivo avaliar o crescimento e a floração da espécie Dendrobium nobile Lindl., sob cinco níveis de luminosidade $\left(83,104,115,154 \mathrm{e} 237 \mu \mathrm{mol} \mathrm{m}^{-2} \mathrm{~s}^{-1}\right)$ propiciadas por subcoberturas providas de telas de sombreamento de $70,50,40,30$ e $0 \%$, respectivamente. Durante 12 meses, as plantas foram irrigadas e adubadas com NPK 10-10-10 e após este período foram avaliadas quanto ao número, comprimento e diâmetro dos pseudobulbos, sendo calculados os incrementos em relação aos dados iniciais. Na época da floração foi contado o número total de gemas, de gemas florais, de gemas vegetativas e de gemas não diferenciadas, registrando-se a antese em cada intensidade luminosa. Utilizou-se o delineamento experimental inteiramente casualizado com cinco tratamentos e sete repetições com duas plantas e as médias foram comparadas pelo teste Tukey a $5 \%$ de probabilidade. Todas as condições de luminosidade foram favoráveis ao cultivo de D. nobile, sendo registrados acréscimos médios de $36,7 \%, 16,0 \%$ e 16,2\% no número, no diâmetro e no comprimento de pseudobulbos, respectivamente. O maior número de gemas florais foi observado na condição de $104 \mu \mathrm{mol} \mathrm{m}^{-2} \mathrm{~s}^{-1}$. D. nobile

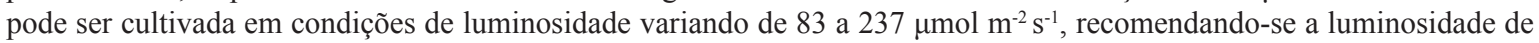
$104 \mu \mathrm{mol} \mathrm{m} \mathrm{m}^{-2} \mathrm{~s}^{-1}$, como forma de promover sua floração.
\end{abstract}

Palavras-chave: Orchidaceae, Floricultura, Sombreamento.

\begin{abstract}
Luminosity on development and flowering of Dendrobium nobile Lindl.

This study, conducted at Jardinocultura area of Faculdade de Ciências Agrárias of UFGD during the period from September of 2010 to August of 2011, had as aim evaluate the cultivation and flowering of Dendrobium nobile Lindl., under five levels of luminosity $\left(83,104,115,154\right.$ e $\left.237 \mu \mathrm{mol} \mathrm{m}^{-2} \mathrm{~s}^{-1}\right)$. During 12 months the plants were irrigated and fertilized with NPK 10-10-10 and after this period they were evaluated for the number, length and diameter of pseudobulbs, being calculated the increments in relation to initial data. At flowering time it was counted the total buds, reproductive buds, vegetative buds and undifferentiated buds and registered the anthesis at each light intensity. The experimental was arranged at completely randomized design with five treatments and seven replicates with two plants and the averages were compared by Tukey test at $5 \%$ probability. All the lighting conditions were favorable to the D. nobile cultivation, being registered increases of $36,7 \%, 16,0 \%$ e $16,2 \%$ in the number, diameter and length of pseudobulbs, respectively. The largest number of reproductive buds was observed at $104 \mu \mathrm{mol} \mathrm{m}^{-2} \mathrm{~s}^{-1}$. D. nobile can be cultivated in the light conditions varying between 83 and $237 \mu \mathrm{mol}$ $\mathrm{m}^{-2} \mathrm{~s}^{-1}$, recommending the luminosity of $104 \mu \mathrm{mol} \mathrm{m} \mathrm{m}^{-2} \mathrm{~s}^{-1}$ to promote their flowering.
\end{abstract}

Keywords: Orchidaceae, Floriculture, Shading.

\section{INTRODUÇÃO}

O mercado de flores brasileiro caracteriza-se, principalmente, pelo baixo consumo per capita, por poucos consumidores assíduos, oferta de produtos tradicionais e concentração de demanda sazonal (JUNQUEIRA e PEETZ, 2008). Segundo os autores, existe uma tendência da descentralização dos pólos produtores, com o objetivo de oferta de produtos mais adaptados às condições regionais, o que implicaria em desenvolvimento econômico e social mais equilibrado em todo o país.

Dentre as famílias botânicas com maior apelo comer- cial estão as plantas das orquidáceas. Dentre os seus atributos ornamentais ressalta-se a durabilidade, perenidade e beleza de suas flores. Além do aspecto ornamental, alguns gêneros ainda fornecem produtos alimentícios, como a baunilha, (espécies do gênero Vanilla), medicinais (como o gênero Dendrobium) e outros produtos utilizados na indústria (HOEHNE, 1949).

O gênero Dendrobium constitui-se em plantas de fácil cultivo, florescendo também em grande escala. Esse gênero tem grande quantidade de espécies e de híbridos, adaptáveis a todos os tipos de clima e, segundo Mattiuz et al. (2006), juntamente com os gêneros Oncidium, Cimbidium,

\footnotetext{
(1) Recebido para publicação em 15/05/2012 e aceito em 06/02/2014;

(2) Professores da Faculdade de Ciências Agrárias (FCA) da Universidade Federal da Grande Dourados (UFGD), Rodovia Dourados - Itahum, km 12 - CEP: 79804-970 Dourados - MS. e-mail: yararosa@ufgd.edu.br

${ }^{(3)}$ Aluna do Programa de Pós - Graduacão em Ensino de Ciências da Universidade Federal de Mato Grosso do Sul (UFMS), Cidade Universitária - CEP. 79070-900 Campo Grande-MS.

(4) Aluna do Programa de Pós-Graduação em Recursos Naturais da Universidade Estadual de Mato Grosso do Sul (UEMS), Rodovia Dourados - Itahum, km 12 - CEP: 79804-970 Dourados - MS.

(5) Técnicas de Laboratório da FCA/UFGD, Rodovia Dourados - Itahum, km 12 - CEP: 79804-970 Dourados - MS.

${ }^{(6)}$ Alunos do Programa de Pós-Graduação em Agronomia da FCA/UFGD, Rodovia Dourados - Itahum, km 12 - CEP: $79804-970$ Dourados - MS.
} 
Phalaenopsis e Cattleya, é um gênero promissor para material de corte.

Dentre suas espécies, Dendrobium nobile Lindl. (conhecida por "olho de boneca") é uma planta herbácea, epífita, perene, entoucerada, originária da China e Himalaia (LORENZI e SOUZA, 2001) sendo amplamente cultivada e comercializada no Brasil, com mais de 15 cultivares que apresentam flores de cores e tamanho variáveis e tolerantes ao frio. A floração, que ocorre preferencialmente no fim do inverno e início da primavera (HUBER, 1994), caracteriza-se pela presença de uma a três flores dispostas nos nós, com sépalas e pétalas brancas, róseas ou amareladas e labelo com manchas roxo-escuras ou claras na garganta (LORENZI e SOUZA, 2001) Figura 1.
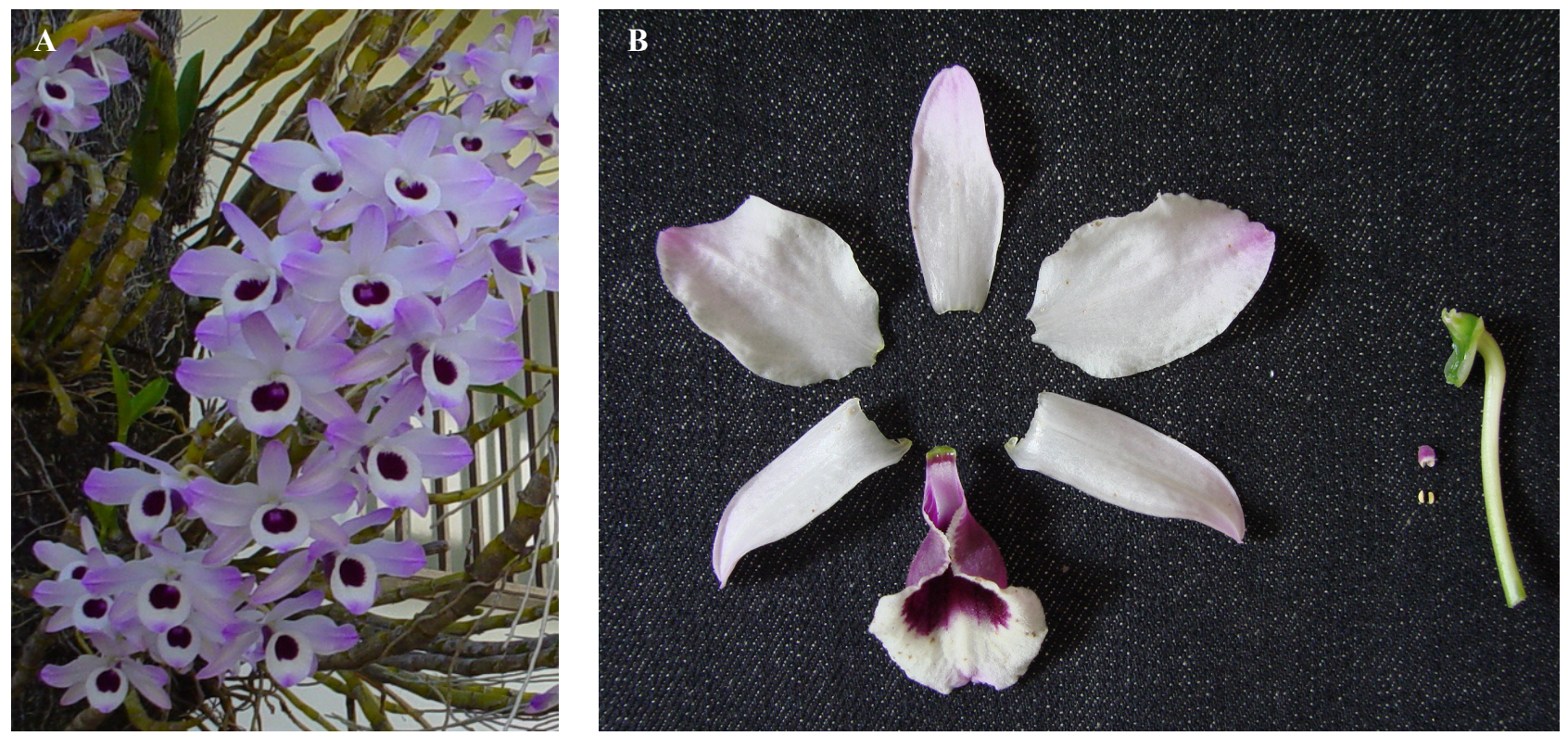

Figura 1. (A) Flores de Dendrobium nobile Lindl.

(B) Partes constituintes das flores de Dendrobium nobile Lindl. UFGD, Dourados, 2011

Figure 1. (A) Dendrobium nobile Lindl flowers.

(B) Constituent parts of flowers of Dendrobium nobile Lindl.. UFGD, Dourados, 2011

No Brasil, a variação de temperatura e umidade relativa observadas nos meses de maio a agosto é propícia a floração de $D$. nobile. Muitos autores salientam que a aclimatização de plântulas é um dos fatores limitantes do cultivo comercial. Kerbauy (2008) relata que, dos fatores abióticos que interferem no desenvolvimento das plantas, a energia luminosa é a que mais influencia o vegetal uma vez que atua sobre a fotossíntese, no fotoperiodismo, na fotomorfogênese e no amadurecimento dos frutos.

Por serem componentes característicos de florestas tropicais úmidas (RICKLEFS, 2003), as espécies epífitas são expostas às baixas intensidades luminosas, uma vez que a luz que atravessa o dossel da floresta é quase toda absorvida pelas folhas das árvores (TAIZ e ZEIGER, 2004), além de sofrer mudanças consideráveis quanto à sua duração e qualidade espectral (FARIAS et al., 1997). Rech et al. (2010) relatam que os maiores incrementos em massa fresca, comprimento e diâmetro de pseudobulbos assim como os maiores comprimentos de sépalas, pétalas, labelo e diâmetro de Dendrobium phalaenopsis var. compactum foram observados em luminosidades de 154 e $237 \mu \mathrm{mol} \mathrm{m} \mathrm{m}^{-2} \mathrm{~s}^{-1}$.

A maioria das orquídeas apresenta danos foliares em presença da radiação solar direta que em cultivos comerciais podem ser atenuados com a utilização de telas de sombreamento, que reduzem a temperatura interna dos abrigos, e vêm sendo apontadas como uma das soluções de menor custo econômico e de grande eficiência (KAMPF, 2000;
MENEZES et al., 2004).

Porém, a baixa intensidade de luz resulta em diminuição da taxa de fotossíntese, na biomassa e na produção, podendo ainda afetar o transporte de fotoassimilados e a relação fonte: dreno (SOUZA et al., 1999). Dessa maneira, alta ou baixa radiação solar pode ser considerada um fator de estresse abiótico, tal como temperatura, disponibilidade de água e nutrição (HE et al., 1998). Assim sendo, o sucesso na adaptação de uma espécie a ambientes com baixa ou alta radiação está associado à eficiência na partição dos fotoassimilados para diferentes partes da planta e na rapidez em ajustar variáveis morfofisiológicas no sentido de maximizar a aquisição dos recursos primários (DIAS FILHO, 1997).

Considerando a importância da luz na produção das orquídeas, este trabalho foi desenvolvido visando a avaliar o cultivo e a floração de Dendrobium nobile Lindl. sob cinco níveis de luminosidade.

\section{MATERIAL E MÉTODOS}

O experimento estudo foi conduzido na área de Jardinocultura da Faculdade de Ciências Agrárias da UFGD, durante o período de setembro de 2010 a agosto de 2011. Foram utilizadas plantas de Dendrobium nobile Lindl. oriundas de propagação vegetativa (keiks) que há dois anos estavam aclimatizadas em viveiro coberto com tela 
de sombreamento de $50 \%$, provido de sistema de irrigação por microaspersão que fornecia lâmina de água equivalente a dois milímetros diários. As plantas estavam plantadas em vasos com capacidade de $300 \mathrm{~mL}$, provido de camada de drenagem de $100 \mathrm{~mL}$ de moinha de carvão lavada, sendo utilizado como substrato a fibra de coco em forma de chips. Setenta plantas foram selecionadas aleatoriamente dentre aquelas que não apresentaram florescimento, sendo posteriormente contado o número de pseudobulbos de cada uma delas assim como medido diâmetro e o comprimento de cada um deles. Os vasos contendo as plantas selecionadas foram alocados sobre bancadas, no interior de viveiro, com tela de $50 \%$ de sombreamento $\left(237 \mu \mathrm{mol} \mathrm{m} \mathrm{m}^{-2} \mathrm{~s}^{-1}\right)$ e cada conjunto de 14 vasos recebeu subcoberturas com telas de $30,40,50$ e $70 \%$ de sombreamento que forneceram intensidades luminosas de $154 ; 115 ; 104$ e $83 \mu \mathrm{mol} \mathrm{m}^{-2} \mathrm{~s}^{-1}$, respectivamente. Um conjunto de 14 vasos não recebeu qualquer tipo de subcobertura ficando exposto apenas à intensidade luminosa do viveiro $\left(237 \mu \mathrm{mol} \mathrm{m}^{-2} \mathrm{~s}^{-1}\right)$.

As plantas permaneceram nestas condições por 12 meses sendo irrigadas três vezes por semana, durante 15 minutos, mediante micro aspersão e adubadas semanalmente, via foliar, com NPK 10-10-10, na concentração de 2,0 mL L-1. Durante este período, as médias das temperaturas e umidades relativas registradas na Estação Meteorológica da FCA foram: temp. $\max .=30,4{ }^{\circ} \mathrm{C}$; temp. $\min =18,8^{\circ} \mathrm{C}$; temp. $\operatorname{med}=23,9^{\circ} \mathrm{C}$; UR $\max =89,8 \%$; UR $\min =45,6 \%$; UR med $=71,5 \%$, precipitação total $=1377,3 \mathrm{~mm}$, velocidade média do vento $=1,5 \mathrm{~m} \mathrm{~s}^{-1}$ e evapotranspiração $=3,3 \mathrm{~mm}$.

Após este período, as plantas foram avaliadas em relação às mesmas características de crescimento e dado o interesse em investigar a hipótese de aumento no número, comprimento e diâmetro dos pseudobulbos em função dos níveis de luminosidade foram calculados os seus percentuais de acréscimos em relação aos valores iniciais, sendo estes resultados considerados para a análise estatística.

Quanto às características de desenvolvimento reprodutivo, foram contados os números de gemas florais, vegetativas (keiks) e gemas não diferenciadas e registradas a data da antese e a durabilidade das flores (número de dias entre a antese e a murcha do mesmo botão floral) em todas as plantas e condições de luminosidade.

Utilizou-se o delineamento experimental inteiramente casualizado com cinco tratamentos e sete repetições com duas plantas. Para análise estatística utilizou-se o aplicativo computacional SISVAR 5.3 (FERREIRA, 2010) e todas as variáveis foram avaliadas mediante análise de variância e posteriormente as médias foram comparadas por meio do teste Tukey até o nível de 5\% de probabilidade.

\section{RESULTADOS E DISCUSSÕES}

Características de crescimento: Não foram observados efeitos significativos $(\mathrm{p}>0,05)$ dos níveis de luminosidade sobre as características de crescimento das plantas (Tabela 1).

Tabela 1. Incrementos no número (INPB), comprimento (ICPB) e diâmetro (IDPB) de pseudobulbos, de Dendrobium nobile Lindl. Observados em função dos níveis de luminosidade. UFGD, Dourados, 2011.

Table 1. Increases in number (INPB), length (ICPB) and diameter (IDPB) of Dendrobium nobile Lindl. pseudobulbs. UFGD, Dourados, 2011.

\begin{tabular}{|c|c|c|c|}
\hline Lum. $\left(\boldsymbol{\mu m o l m} \mathbf{m}^{-\mathbf{- 1}}\right)$ & INPB (\%) & ICPB (\%) & IDPB (\%) \\
\hline 237 & $48,99 \mathrm{a}$ & $8,50 \mathrm{a}$ & $12,74 \mathrm{a}$ \\
\hline 154 & $35,52 \mathrm{a}$ & $10,86 \mathrm{a}$ & $10,40 \mathrm{a}$ \\
\hline 115 & $43,05 \mathrm{a}$ & $12,26 \mathrm{a}$ & $21,05 \mathrm{a}$ \\
\hline 104 & $24,90 \mathrm{a}$ & $23,25 \mathrm{a}$ & $24,42 \mathrm{a}$ \\
\hline 83 & $31,12 \mathrm{a}$ & $25,38 \mathrm{a}$ & $12,78 \mathrm{a}$ \\
\hline CV(\%) & 17,37 & 12,47 & 12,15 \\
\hline Média & 36,72 & 16,05 & 16,28 \\
\hline
\end{tabular}

Médias seguidas de mesma letra, na coluna, não diferem entre si (Tukey 5\% de probabilidade)

Nenhuma das condições de luminosidade pode ser considerada estressora ao crescimento de $D$. nobile, pois houve acréscimos positivos em todas as características analisadas (Tabela 1). Os maiores acréscimos médios foram observados em relação ao número de pseudobulbos $(36,72 \%)$, seguido dos incrementos em diâmetro $(16,28 \%)$ e altura $(16,05 \%)$. Os resultados foram promissores quer para a produção de flores quer para a produção de princípios ativos com propriedades farmacológicas.

Como os pseudobulbos são órgãos de reserva de fotoassimilados e de água, quanto maiores em número, comprimento e diâmetro, maior será a resistência e a sobrevivência das plantas em condições de estresse hídrico e nutricional e melhor será a qualidade das flores geradas (ASSIS et al., 2003). Além disso, o incremento no número de pseudobulbos produzidos é extremamente vantajoso sob o ponto de vista ornamental, uma vez que a espécie floresce com maior abundância nos pseudobulbos de um ano.

Os incrementos no número, altura e diâmetro de pseudobulbos também são vantajosos quando a planta é explorada com finalidade farmacológica. Segundo Lin et al. (2003), D. nobile apresenta propriedades antioxidantes, vasodilatadoras e anticancerígenas que são encontradas em princípios ativos extraídos de seus pseudobulbos. Assim, quanto maior o número, o diâmetro e comprimento destas estruturas, maior o potencial de produção dos compostos. 
Características de desenvolvimento: Não foram observados efeitos significativos $(p>0,05)$ dos níveis de luminosidade sobre o número total de gemas, de gemas ve- getativas e de gemas não diferenciadas que apresentaram, respectivamente, valores médios de 2,28; 0,11 e 1,11 gemas por planta (Tabela 2).

Tabela 2. Número total de gemas (NG), de gemas florais (GF), de gemas vegetativas (GV) e de gemas não diferenciadas (GND), tempo para antese (AT) e durabilidade das flores (DF) de Dendrobium nobile Lindl. UFGD, Dourados, 2011.

Table 2. Total buds (NG), reproduductive buds $(G F)$, vegetative buds $(G V)$ and indiferenciate buds (GND), time to anthesis (AT) and durability of flowers (DF) of Dendrobium nobile Lindl. UFGD, Dourados, 2011.

\begin{tabular}{|c|c|c|c|c|c|c|}
\hline $\begin{array}{c}\text { I.Lum. } \\
\left(\mu \mathrm{molm}^{-2} \mathrm{~s}^{-1}\right)\end{array}$ & NG & GF & GV & GND & $\begin{array}{c}\text { AT } \\
(\text { dias })\end{array}$ & DF \\
\hline 237 & $2,71 \mathrm{a}$ & $0,71 \mathrm{~b}$ & $0,28 \mathrm{a}$ & $1,71 \mathrm{a}$ & $362,00 \mathrm{a}$ & $22,00 \mathrm{~b}$ \\
\hline 154 & $1,85 \mathrm{a}$ & $0,71 \mathrm{~b}$ & $0,00 \mathrm{a}$ & $1,14 \mathrm{a}$ & $361,86 \mathrm{a}$ & $18,00 \mathrm{c}$ \\
\hline 115 & $1,57 \mathrm{a}$ & $0,57 \mathrm{~b}$ & $0,14 \mathrm{a}$ & $0,85 \mathrm{a}$ & $357,10 \mathrm{~b}$ & $22,00 \mathrm{~b}$ \\
\hline 104 & $4,14 \mathrm{a}$ & $3,00 \mathrm{a}$ & $0,14 \mathrm{a}$ & $1,00 \mathrm{a}$ & $357,00 \mathrm{~b}$ & $26,00 \mathrm{a}$ \\
\hline 83 & $1,14 \mathrm{a}$ & $0,42 \mathrm{~b}$ & $0,00 \mathrm{a}$ & $0,85 \mathrm{a}$ & $357,00 \mathrm{~b}$ & $26,00 \mathrm{a}$ \\
\hline CV $(\%)$ & 31,56 & 30,07 & 12,79 & 32,47 & 0,30 & 2,71 \\
\hline Média & 2,28 & 1,08 & 0,11 & 1,11 & 358,99 & 22,51 \\
\hline
\end{tabular}

Médias seguidas de mesma letra, na coluna, não diferem entre si (Tukey $5 \%$ de probabilidade)

Intensidade luminosa de $104 \mu \mathrm{mol} \mathrm{m}^{-2} \mathrm{~s}^{-1}$ foi a que propiciou maior número de gemas florais por planta (Tabela 2 ), sendo seus valores seis vezes superiores aos registrados em condições de 115 e 83 e quatro vezes maiores aos registrados em condições de 237 e $154 \mu \mathrm{mol} \mathrm{m}^{-2} \mathrm{~s}^{-1}$.

Intensidades luminosas iguais ou inferiores a $104 \mu \mathrm{mol}$ $\mathrm{m}^{-2} \mathrm{~s}^{-1}$ propiciaram duração média da floração de 26 dias, enquanto os valores superiores propiciaram redução da duração da floração em pelo menos quatro dias (Tabela 2).

A antese também foi influenciada $(\mathrm{p}<0,05)$ pelas intensidades luminosas. Valores iguais ou inferiores a $115 \mu \mathrm{mol}$ $\mathrm{m}^{-2} \mathrm{~s}^{-1} \mathrm{a}$ anteciparam em aproximadamente cinco dias em relação às intensidades de 154 e $237 \mu \mathrm{mol} \mathrm{m} \mathrm{m}^{-2} \mathrm{~s}^{-1}$ (Tabela 2). Os resultados encontrados neste trabalho contrapõem-se aos de Macedo et al. (2011) que, estudando o comportamento de um híbrido de denfal, verificaram atraso na antese à medida que a intensidade luminosa diminuiu de 237 para $83 \mu \mathrm{mol} \mathrm{m} \mathrm{m}^{-2} \mathrm{~s}^{-1}$ indicando que espécies de um mesmo gênero comportam-se de maneiras distintas frente a um mesmo estímulo, evidenciando assim a necessidade de pesquisas específicas em relação ao material vegetal que se deseja cultivar. Segundo Cardoso (2007), pouco se conhece a respeito dos fatores bióticos e abióticos que influenciam a floração desta família botânica uma vez que a identificação dos mesmos é dificultada devido ao grande número de espécies, distribuídas em diferentes habitats caracterizados por condições ambientais distintas.

Os valores percentuais dos incrementos no número, comprimento e diâmetro de pseudobulbos assim como o número total de gemas e o de gemas florais são apresentados na Figura 2.

$\mathrm{O}$ incremento positivo no número de pseudobulbos em todas as condições de luminosidade estudadas (Figura 2) é vantajoso tanto para a exploração comercial de flores quanto para a de fármacos. Bernardi et al. (2004) relatam que em plantas de $D$. nobile a quantidade de flores determina seu valor comercial e o número de flores produzidas está diretamente relacionado ao comprimento do pseudobulbo, e ao seu número.

Considerando o relato destes autores e os resultados observados neste trabalho, após o perfilhamento, as plantas devem ser submetidas à luminosidade de $104 \mu \mathrm{mol} \mathrm{m} \mathrm{m}^{-2}$ $\mathrm{s}^{-1}$, pois foi a condição luminosa que apresentou o melhor equilíbrio entre os incrementos da parte aérea das plantas (Figura 2), além de propiciar o maior número de gemas e de gemas florais. 


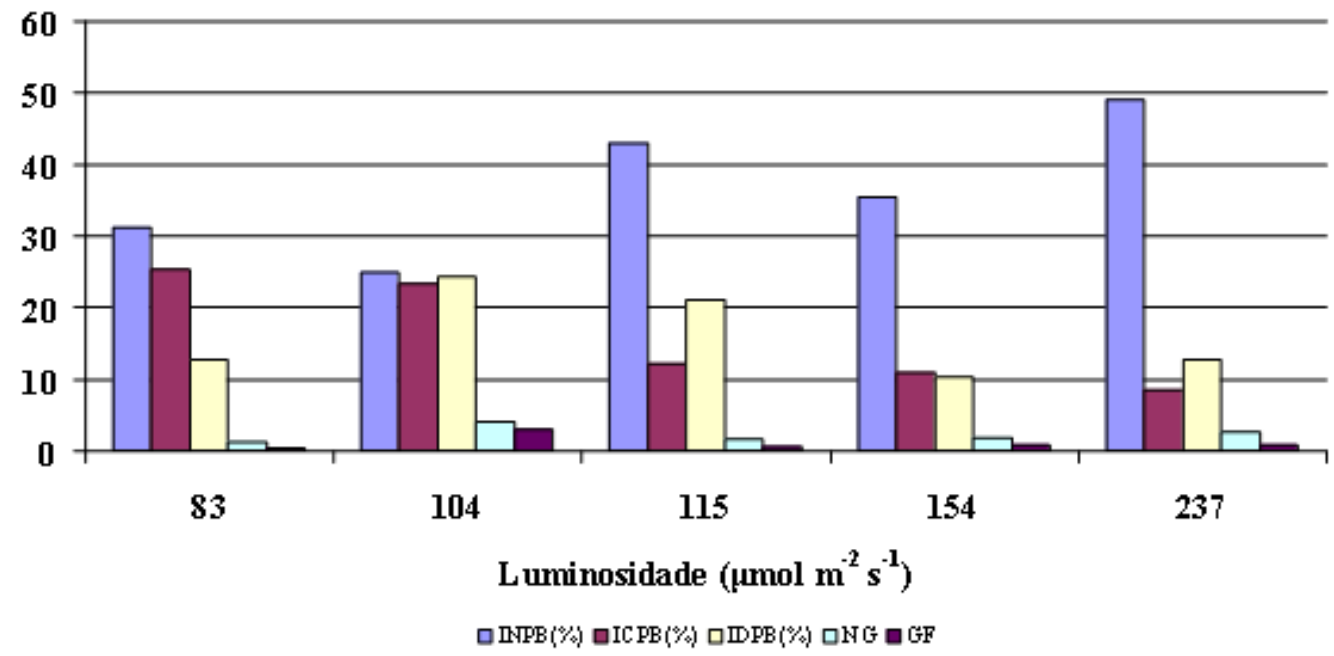

Figura 2. Incrementos no número (INPB), comprimento (ICPB) e diâmetro (IDBP) de pseudobulbos, número total de gemas (NG) e de gemas florais (GF) de Dendrobium nobile Lindl. UFGD, Dourados, 2011

Figure 2. Increases in number (INPB), length (ICPB) and diameter (IDPB) of pseudobulbs, total (NG) and reproductive (GF) buds of Dendrobium nobile Lindl. UFGD, Dourados, 2011

\section{CONCLUSÕES}

Dendrobium nobile Lindl pode ser cultivada em condições de luminosidade variando de 83 a $237 \mu \mathrm{mol} \mathrm{m} \mathrm{m}^{-2} \mathrm{~s}^{-1}$, recomendando-se a luminosidade de $104 \mu \mathrm{mol} \mathrm{m} \mathrm{m}^{-2} \mathrm{~s}^{-1}$ como forma de promover sua floração.

\section{AGRADECIMENTOS}

À UFGD, ao CNPq e à FUNDECT pela concessão das bolsas de Iniciação Científica e financiamentos concedidos.

\section{REFERÊNCIAS}

ASSIS, A. M.; COLOMBO, L. A.; FARIA, R. T.; FONSECA, I. C. B. Longevidade pós-colheita de pseudobulbos com flores de Dendrobium nobile (Orchidaceae). Revista Brasileira de Horticultura Ornamental, v. 9, n. 1, p. 85$87,2003$.

BERNARDI, A. C.; FARIA, R. T.; CARVALHO, J. F. R. P.; UNEMOTO, L. K.; ASSIS, A.M. Desenvolvimento vegetativo de Dendrobium nobile Lindl. fertirrigadas com diferentes concentrações da solução nutritiva de Sarruge. Semina v. 25, p. 13-20, 2004.

CARDOSO, J. C. 2007. Ácido giberélico (GA, ) na indução do florescimento de orquídeas. Botucatu: Universidade Estadual Paulista, 2007. 50p. Dissertação (Mestrado em Fisiologia Vegetal).

DIAS-FILHO, M. B. Physiological response of Solanum crinitum Lam. to contrasting light environments. Pesquisa Agropecuária Brasileira, Brasília, v. 32, n. 8, p. 789-796, 1997.
FARIAS, V. C. C.; VARELA, V. P.; COSTA, S. S.; BATALHA, L. F. Análise de crescimento de mudas de cedrorana (Cedrelinga catenaeformis (Ducke) Ducke) cultivadas em condições de sombreamento. Revista Brasileira de Sementes, Brasília, v. 19, n. 2, p. 193-200, 1997.

FERREIRA D. F. 2010. Programa de análises estatísticas (Statistical Analysis Software) e planejamento de Experimentos - SISVAR 5.3. Universidade Federal de Lavras.

HE, J.; KHOO, G. H.; HEW, C. S. Susceptibility of CAM Dendrobium leaves and flowers to high light and high temperature under natural tropical conditions. Environmental and Experimental Botany, v. 40, n. 3, p. 255264, 1998.

HOEHNE, F. C. Iconografia de Orchidaceas do Brasil. Instituto de Botânica de São Paulo. 1949, 601 p.

JUNQUEIRA, A. H.; PETTZ, M. da S. Mercado interno para os produtos da floricultura brasileira: características, tendências e importância sócioeconômica recente. Revista Brasileira de Horticultura Ornamental, v. 14, n. 1, p. 37-52, 2008.

KAMPF, A. N. Produção comercial de plantas ornamentais. Guaíba: Agropecuária, 2000, 254p.

KERBAUY, G. B. Fisiologia vegetal. 2 ed. Rio de Janeiro: Guanabara, 2004, 431p.il.

LIN, P.; BI, Z.; XU, H.; WANG, Z.; XU, L. Advances in studies on pharmacology of plants from Dendrobium Sw. Chinese Traditional and Herbal Drugs, v. 34, p. 19-22, 2003. 
LORENZI, H.; SOUZA, H. M. Plantas ornamentais no Brasil: arbustivas, herbáceas e trepadeiras. Nova Odessa, SP: Plantarum, p. 833-837, 2001.

MACEDO, M. C.; ROSA, Y. B. C J.; SCALON, S. de P. Q.; ROSA JUNIOR, E. J.; VIEIRA, M. do C.; TATARA, M.B. Substratos e intensidades de luz no cultivo de orquídea denfal. Horticultura Brasileira, v. 29, n. 2, p. 168$173,2011$.

MATTIUZ, C. F. M.; RODRIGUES, T. J. D.; MATTIUZ, B. Aspectos fisiológicos de orquídeas cortadas. Revista Brasileira de Horticultura Ornamental, v. 12, p. 21-30, 2006.

MENEZES, N. L.; FRANZINI, S. M.; ROVERSI, T.; NUNES, E. P. Germinação de sementes de Salvia splendens Sellow em diferentes temperaturas e qualidade de luz. Revista Brasileira de Sementes, v. 26, p. 32-37, 2004.

RECH, A. R.; ROSA, Y. B. C. J.; SILVA, H. M. Comportamento de dendróbio borboleta (Dendrobium phalaenopsis var. compactum C.T. White - Orchidaceae) sob diferentes níveis de sombreamento. Revista Agrarian, v. 3, n. 7, p. 84-87, 2010.
RICKLEFS, R. E. A economia da natureza. Rio de Janeiro: Guanabara Koogan, 2003, 503p.

SOUZA, J. R. P.; MEHL, R.O.; RODRIGUES, J.D.; PEDRAS, J. F. Sombreamento e o desenvolvimento e produção de rabanete. Scientia Agrícola, Piracicaba, v. 56, n. 4, p. 987-992, 1999.

TAIZ. L.; ZEIGER, E. Fisiologia vegetal. Porto Alegre: Artmed Editora, 2004, 719p.

TREMBLAY, R. L.; ACKERMAN, J. D.; ZIMMERMAN, J. K.; CALVO, R. N. Variation in sexual reproduction in orchids and its evolutionary consequences: a spasmodic journey to diversification. Biological Journal of the Linnean Society. v. 84, p. 1-54, 2005. 